Mots. Les langages du politique

$94 \mid 2010$

Trente ans d'étude des langages du politique

(1980-2010)

\title{
Argumentation-rhétorique. Les eaux mêlées
}

\section{Christian Plantin}

\section{(2) OpenEdition \\ Journals}

Édition électronique

URL : https://journals.openedition.org/mots/19856

DOI : $10.4000 /$ mots. 19856

ISSN : 1960-6001

Éditeur

ENS Éditions

\section{Édition imprimée}

Date de publication : 30 novembre 2010

Pagination : 23-30

ISBN : 978-2-84788-235-3

ISSN : 0243-6450

Référence électronique

Christian Plantin, «Argumentation-rhétorique. Les eaux mêlées », Mots. Les langages du politique [En ligne], 94 | 2010, mis en ligne le 06 novembre 2012, consulté le 28 avril 2022. URL : http:// journals.openedition.org/mots/19856; DOI : https://doi.org/10.4000/mots.19856 


\section{Mots \\ Les langages du politique}

№ 94 novembre 2010

\section{Trente ans d'étude des langages du politique (1980-2010)}

ouvrage coordonné par Paul BACOT, Marlène Coulomb-GulLY, Jean-Paul Honoré, Christian Le BART, Claire Oger, Christian Plantin

\section{SOMMAIRE}

Paul Bacot, Marlène Coulomb-Gully, Jean-Paul Honoré, Christian Le Bart, Claire Oger, Christian Plantin

Le discours politique n'est pas transparent. Permanence

et transformations d'un objet de recherche

OUTILS ET ENJEUX DU DISCOURS POLITIQUE

Ruth Amossy, Roselyne Koren

Argumentation et discours politique

Christian Plantin

Argumentation-rhétorique. Les eaux mêlées

Caroline Ollivier-Yaniv

Discours politiques, propagande, communication, manipulation 
Marc Bonhomme

La caricature politique

Paul Bacot

Développement et diversification d'une onomastique politique

Ruth Wodak

The Discursive Construction of History. Brief Considerations

Henri Boyer

Les politiques linguistiques

\section{LIEUX DE LA PRODUCTION DU DISCOURS POLITIQUE}

Christian Le Bart

Parler en politique

Dominique Maingueneau

Le discours politique et son «environnement»

Alice Krieg-Planque, Claire Oger

Discours institutionnels. Perspectives pour les sciences

de la communication

Sophie Béroud, Josette Lefèvre

Le corpus syndical. Une expérience au long cours

Corinne Gobin, Jean-Claude Deroubaix

L'analyse du discours des organisations internationales.

Un vaste champ encore peu exploré

François de la Bretèque

Le retour de la parole politique dans le cinéma français

Jean-Claude Soulages

Vie et mort du citoyen cathodique

Jacques Guilhaumou

Les discours de la Révolution française.

Aperçu d'ensemble d'un trajet de recherche (1980-2009) 


\section{DISCIPLINES ET CHAMPS DE RECHERCHE POUR L'ÉTUDE DES LANGAGES DU POLITIQUE}

Philippe Braud

L'apport de la science politique à l'étude des langages du politique

Claire Blandin

L'apport de l'histoire des médias à l'étude des langages du politique

Jean-François Tétu, Bernard Lamizet

Les SIC et les langages du politique

Sylvianne Rémi-Giraud

Sémantique lexicale et langages du politique.

Le paradoxe d'un mariage difficile?

Marlène Coulomb-Gully, Juliette Rennes

Genre, politique et analyse du discours.

Une tradition épistémologique française gender blind

Johannes Angermüller

Analyser le discours politique en Allemagne (1980-2010)

Érik Neveu

L'apport de Pierre Bourdieu à l'analyse du discours.

D'un cadre théorique à des recherches empiriques

Roselyne Ringoot

Questionner le discours avec Michel Foucault.

Actualisations théoriques et actualité éditoriale

\section{ENTRETIEN}

Maurice Tournier

Mots et politique, avant et autour de 1980

La revue Mots. Les langages du politique encourage l'usage des rectifications de l'orthographe proposées par le Conseil supérieur de la langue française et approuvées par l’Académie (Journal officiel, $n^{\circ}$ 100, 6 décembre 1990). 


\section{Christian Plantin}

\section{Argumentation-rhétorique. Les eaux mêlées}

La tradition rhétorique argumentative s'intéresse aux ensembles discursifs produits dans des situations où les opinions divergent. Ces discours sont orientés par des questions telles que : «Est-il vraiment coupable?», «Devons-nous instaurer une taxe carbone? » Ces questions sont dites «disputées» dans la mesure où elles reçoivent des réponses incompatibles, nécessairement accompagnées de justifications plus ou moins appropriées.

Le discours politique n'est pas le seul objet de l'analyse argumentative classique. Le discours du tribunal est souvent pris comme prototype du discours argumentatif, dans la mesure où s'y incarnent de façon évidente les trois pôles discursifs caractéristiques de la situation argumentative (Proposant, Opposant, Tiers, en l'occurrence Accusateur, Défenseur, Juge).

L'argumentation est souvent définie fonctionnellement par l'effet de persuasion qui lui serait associé ; le discours de propagande (religieux, politique, idéologique) est le domaine où parler de croyance, d'adhésion des esprits, d'assentiment fait le plus sens, où le travail de persuasion s'effectue dans sa double dimension, persuasive et consensuelle (édifier, renforcer l'adhésion des fidèles et des adhérents à un commun programme et à un commun credo) comme dissensuelle et apologétique (défier les opposants et les infidèles). L'argumentation rhétorique est un cadre théorique dans lequel a été pensé le discours politique depuis plus de deux millénaires. Parmi les acquis les plus robustes de cette réflexion, on pourrait citer les travaux sur les topoï du discours politique, c'est-à-dire des mini-discours relativement abstraits, considérés comme porteurs de «bonnes raisons » et adaptables à toutes les circonstances, comme l'ont découvert, redécouvert ou rappelé Aristote, Bentham, Schopenhauer ou Hirschman. 


\section{Deux traditions américaines d'analyse rhétorique-argumentative du discours politique}

Si l'on cherche à caractériser la situation de l'analyse rhétorique du discours politique au cours de ces trente dernières années, il faut d'abord rappeler une vérité incontournable : c'est une discipline en soi aux États-Unis et dans les pays qui adoptent leur mode d'organisation académique. Il faut ensuite s'interroger sur les orientations des études d'argumentation, à la fois dans leur dynamique propre et en rapport avec l'étude du discours.

\section{Analyse rhétorique du discours politique [Rhetorical analysis of public address]}

Les États-Unis entretiennent en effet une tradition d'étude rhétorique du discours politique, ininterrompue depuis l'époque coloniale, appuyée sur une solide infrastructure académique et un nombre impressionnant de supports de publications.

Elle est prise en charge par les Rhetorical Studies, implantées dans des Speech and Communication Departments, dont l'origine remonte au tournant des $19^{\mathrm{e}}-20^{\mathrm{e}}$ siècles. L'ambiance générale de ces études demeure étrangère au public et à l'esprit français. Elles reposent sur une vision de l'histoire et du destin américains comme scandés, voire déterminés par la prise de parole publique. Les performances oratoires d'Obama sont un produit de cette culture.

La méthode d'étude dominante au milieu du siècle dernier était dite néoclassique. Elle ambitionnait la création d'une critique rhétorique [rhetorical criticism] qui serait la contrepartie, pour le discours sociopolitique, de ce qu'est la critique littéraire [literary criticism] pour le discours poétique, dramatique ou fictionnel.

Ces études reposent sur des corpus de discours recueillis dans des anthologies comme An American Primer (Boorstin, 1968) ou les trois volumes des Great Issues in American History (Hofstadter, 1958-1969) qui rassemblent de grands discours qui ont marqué l'histoire américaine.

En gros, la façon de faire est la suivante. On précise le sens de «l'occasion », le contexte et l'auditoire pour montrer comment et dans quelle mesure la situation a été transformée par le discours. L'analyse repose sur un «cercle rhétorique » : elle considère successivement chacun des pas qui, selon la tradition scolaire, permettent de produire le discours : quels sont les arguments, comment sont-ils composés, quelle est la figure incarnée par l'orateur, quels effets de style et d'éloquence a-t-il utilisés, a-t-il remué émotionnellemenet son auditoire, etc. (Hochmuth, 1955). Cette méthode a été fortement critiquée dans les années soixante (Black, 1965); on lui reproche notamment d'avoir engendré 
une sous-littérature scientifique ressassant sans imagination et à tout propos un peu de logos, un peu de pathos, un peu d'ethos. Le point essentiel est peutêtre le suivant. Cette théorie repose sur une conception, toujours implicite, d'un sujet parlant, démiurge discursif, maître de ses intentions et de ses choix (d'arguments, de style), contrôlant stratégiquement son image, calmant et apaisant les passions, déterminant et non déterminé par son discours, bref un sujet discursif plein, «moderne », que tout oppose au sujet dispersé " postmoderne».

Ce courant, redéfini par des propositions sur l'étude des rhétoriques des mouvements, l'élargissement de ses thèmes à la rhétorique du non verbal, des rhétoriques de la narration et des solidarités imaginaires [fantasy theme analysis], l'étude des formes rhétoriques non conventionnelles [disruptive rhetoric], et influencé par les visions dialectiques, se retrouve bien présent dans les mouvements récents d'analyse de l'argumentation rhétorique.

D’une façon générale, et comme il est normal, les études rhétoriques du discours politique se sont rapprochées des études de communication : l'orateur se métamorphose en énonciateur et en voix, l'auditoire en public et en cible, l'ethos en image de soi, des autres et du produit à vendre, etc.

\section{L'argumentation comme discipline critique [Critical thinking]}

Outre-Atlantique, une seconde source des études d'argumentation, sans relation originelle avec la précédente, se situe non plus dans les Speech Departments, mais dans les départements de philosophie. Ce courant autonome trouve ses fondements théoriques dans un ouvrage savant sur l'histoire de la notion de paralogisme (au sens large), Fallacies (Hamblin, 1970), et veut, sur cette base adaptée, répondre à de nouveaux impératifs pédagogiques apparus dans les années soixante-dix. Dans le cahier des charges des départements de philosophie figure en effet l'apprentissage de la pensée critique [critical thinking]; pour s'acquitter de cette tâche, les enseignants développaient ce cursus à partir d'une initiation à la logique élémentaire. On espérait plus ou moins explicitement par ce biais «apprendre à raisonner», prétention tout à fait classique puisque la logique, au moins la logique classique, se présente comme un «art de penser».

Or cet enseignement fut un échec total. Kahane a donné de cet échec une description qui a fait date : «La plupart des étudiants souhaitent aujourd'hui un cours traitant du raisonnement quotidien, un cours traitant des argumentations qu'ils entendent et lisent à propos du racisme, de la pollution, de la pauvreté, du sexe, de la guerre atomique, de l'explosion démographique et, en général, de tous les problèmes auxquels se trouve confrontée l'humanité en cette seconde moitié du $20^{\circ}$ siècle.» (Kahane, 1971 p.7). Il est caractéristique que toute la demande telle que la rapporte Kahane porte sur la critique du discours politique dominant. 
Les choses ont évidemment évolué en quarante ans. Néanmoins, c'est ce courant, dit de «logique informelle», développé notamment au Canada autour de la revue Informal Logic (Blair, Johnson), qui a, à la suite de Hamblin, orienté l'étude de l'argumentation vers l'étude des conditions de validité des argumentations ordinaires en se fondant sur la notion de fallacie. En France, où la recherche a pris d'autres directions, le terme et la problématique des fallacies, il est vrai parfois appliqués de façon surprenante, sont ignorés lorsqu'il ne sont pas exécrés. Mais la question qui reste est celle de l'éristique politique ou, à l'inverse, celle de savoir si, et comment, on peut définir le plus objectivement possible ce qu'est un discours - entre autres un discours politique fallacieux. C'est le courant de logique informelle qui a réintroduit dans l'argumentation une dimension critique absente des modèles néoclassiques de la rhétorique argumentative.

\section{La rencontre argumentation-rhétorique}

La situation de la recherche dans les vingt dernières années est marquée par la rencontre entre ces deux courants. Du côté européen, l'International Society for the Study of Argumentation a joué un rôle essentiel dans la «mondialisation » des études d'argumentation. Cette société, fondée en 1986 par Frans van Eemeren et Rob Grootendorst, a tenu sa première conférence internationale à Amsterdam, également en 1986. Les Proceedings, édités par Frans van Eemeren et Rob Grootendorst, Charles Arthur Willard et J. Anthony Blair, sont parus en 1987. Cette initiative institutionnelle et scientifique a marqué un moment décisif dans la structuration de la communauté mixte des études de rhétorique et d'argumentation. Depuis un quart de siècle, les conférences internationales se tiennent tous les quatre ans, régulièrement accompagnées d'imposants volumes de Proceedings. Les six séries de Proceedings actuellement parus constituent un corpus qu'il est légitime de considérer comme représentatif de ce qui se fait en argumentation-rhétorique et où l'on trouvera, entre autres, des études du discours politique.

1986 est certainement la date où se sont rencontrés, côtoyés et parfois mélangés des courants d'études de l'argumentation relevant des sources déjà mentionnées. Ces nouvelles tendances se retrouvent dans des revues comme Argumentation ou, en français, dans la toute nouvelle revue en ligne Argumentation et Analyse du discours ${ }^{1}$.

À l'heure actuelle, l'analyse rhétorique-communicationnelle du discours politique est un des thèmes essentiels des travaux publiés dans des revues comme celles citées ci-dessous en note (la liste est très loin d'être exhaustive).

1. URL : [http://aad.revues.org/]. Site consulté le 22 juillet 2010. 
Certaines de ces revues se rattachent explicitement à la rhétorique ${ }^{2}$, d'autres plus généralement à l'étude de la parole [speech]3 ou de l'argumentation 4 ; dans d'autres encore, la recherche rhétorique se fond dans l'étude de la communication5. Une bibliographie des études du langage politique d'inspiration rhétorique prendrait plusieurs volumes. La recherche francophone est très peu présente, pour ne pas dire totalement absente de ces revues.

\section{Situation des études d’argumentation. La vie après le revival}

Dans une tout autre orientation, le destin de l'analyse argumentative du discours politique est évidemment lié de quelque manière à celui d'autres approches de l'argumentation, engagées dans d'autres domaines (argumentation et acquisition du savoir, argumentation et débats citoyens) ou dans d'autres préoccupations (par exemple, dégager des modèles logiques, des modèles rationnels, des modèles linguistiques... de l'activité argumentative).

La situation peut être schématisée - de manière outrageusement sybilline - comme suit. D'une façon générale, les études d'argumentation ont pris un nouveau départ après la seconde guerre mondiale (la fameuse période du revival) dans différentes directions, bien distinctes à leur origine : un néoclassicisme (Perelman, Olbrechts-Tyteca); des logiques appliquées au discours et au raisonnement naturels (Toulmin, Grize, Blair et Johnson; logiques substantielle, logique naturelle, logique informelle); une linguistique de l'argumentation (Ducrot); des théories logiques ou pragmatiques des fallacies (Hamblin, Woods et Walton); des approches dialectiques (Van Eemeren et Grootendorst) ou interactionnelles et dialogales.

Les trente années qui viennent de s'écouler sont ainsi caractérisées par la multiplication des styles de travail en argumentation et, depuis une quinzaine d'années, l'apparition d'orientations plus éclectiques, « transcourants». Cette diversité constitue une richesse, mais il reste évident que la question de l'articulation globale de ces recherches reste posée et que cela a des retentissements dans tous les domaines. Il peut être utile d'exposer quelques effets négatifs - on excusera l'omission diplomatique des références. Il n'est pas rare de voir de respectables concepts fondamentaux redéfinis de façon arbitraire ou carrément fantaisiste, utilisés de manière ad hoc ou simplement évoqués

2. Philosophy and Rhetoric; Rhetoric \& Public Affairs; Rhetoric Review; Rhetorica, etc.

3. Quarterly Journal of Speech; Southern Speech Communication Journal; Southern Speech Journal; Western Journal of Speech Communication; Central State Speech Journal; Speech Monographs; Speaker and Gavel, etc.

4. Argumentation and Advocacy.

5. Communication Monographs; Communication Quarterly; Communication studies; Journal of Applied Communication Research; Journal of Communication; The Southern Communication Journal, etc. 
pour leur effet décoratif. Prenons par exemple la question des formes de raisonnement langagiers ordinaires, c'est-à-dire celle des types et des formes d'arguments; c'est une question classique, bien documentée, clairement et fructueusement discutée dans différents cadres théoriques. Or on trouve des recherches qui se soucient peu de cet état de l'art, dont elles semblent même ignorer l'existence.

De même, on constate des effets de mode : alors que jusqu'au milieu des années quatre-vingt-dix, tout le monde cherchait son salut dans la théorie de l'argumentation dans la langue, il s'est effectué depuis un basculement vers un «tout Perelman » aussi surprenant; certaines recherches appliquées, toutes acquises à un paradigme, se privent des ressources pertinentes pour leur problèmatiques qu'elles trouveraient chez leur voisin (et à la même enseigne). Il en résulte une impression d'argumentation self-service où différents items sont exploités sur le mode fire and forget. Il s'ensuit que les bons esprits, qui sont des esprits prudents, affichent légitimement une attitude aussi révérencieuse que distante vis-à-vis d'un domaine aussi désordonné.

Mauvais esprit mis à part, il y a là un problème. Ce n'est pas faute de manuels d'introduction : il en existe beaucoup, tous excellents; aucun ne dispense de jeter un coup d'œil sur les textes originaux. Plus profondément, la faille essentielle semble être le vide de la formation académique; on ne connaît que les (auto)formations express, on manque de cursus systématique en argumentation en langue française.

Mais on pourrait prendre la question par un autre biais, celui de l'intégration de ce type d'approche à celui des cadres de l'analyse du discours. Le problème est le suivant.

Alors qu'ils ont rencontré naturellement et immédiatement les travaux menés dans les Speech Departments autour de l'analyse du discours sociopolitique, les travaux de Perelman étaient loin d'être au centre des grands mouvements qui ont transformé les sciences humaines dans les années soixante-dix. La distance entre approche à la française et approche rhétorique du discours politique a été dépassée sans être vraiment pensée.

On peut mesurer cette distance à quelques indices. D'une part, peu de temps avant la naissance de Mots. Les langages du politique, il était dit des travaux de Perelman et Olbrechts-Tyteca qu'ils «se situ[aient] en marge de la plupart des reprises modernes de la rhétorique» (Lacoste, 1970, p. 235). Cette affirmation était profondément vraie en 1970, étant entendu que par « reprises modernes de la rhétorique », on renvoie aux théories des figures développées dans le cadre d'une théorie linguistique d'inspiration structuraliste.

Sur un autre versant, Nouvelle rhétorique et analyse du discours «à la française » étaient des contemporains qui se tournaient le dos. Dans Les Vérités de La Palice, Michel Pêcheux définit une formation discursive comme «ce qui peut et doit être dit (articulé sous la forme d'une harangue, d'un sermon, d'un pam- 
phlet, d'un exposé, d'un programme, etc.), dans une formation idéologique définie, c'est-à-dire à partir d'une position de classe au sein d'une conjoncture donnée» (Maldidier, 1990, p. 44). Les exemples donnés de « formes articulées» de discours sont toutes des genres rhétoriques classiques, dont l'étude, à la manière de Perelman, pourrait très bien être menée dans un programme de Speech Department.

Mais discours a bien des sens. La notion de discours telle qu'elle était définie par Michel Foucault ou dans l'ouvrage fondateur de Dominique Maingueneau (1976) n'avait rien à voir avec la notion de discours comme public address, comme disent les Anglo-Saxons (voir supra, première partie de cet article), c'est-à-dire «ce qui, dit en public, traite d'un sujet avec une certaine méthode et une certaine longueur » (Littré). Cette acception du terme discours ne figure pas parmi les six acceptions distinguées par Maingueneau (1976, p.11-12); les mondes théoriques sont incommensurables.

\section{Pour une restructuration critique des études d'argumentation}

Un pedigree politique impeccable, un produit déjà mondialisé, un côté pluridisciplinaire qui convient à l'époque, des orientations théoriques substantielles et débattables (autochtones et transatlantiques), des états de la question fournis, un enseignement à structurer - et quelques bonnes polémiques à revivifier. Il semble donc que toutes les conditions sont réunies pour que s'épanouisse une école française d'analyse rhétorique-argumentative du discours politique. Mais cela ne se fera pas sans l'institutionnalisation, quelque part, d'une formation ouverte à la recherche en argumentation.

\section{Références}

ARISTOTE, 1932, Rhétorique, trad. M. Dufour, Paris, Les Belles-Lettres.

Bentham J., 1998 [1824], Fragment sur le gouvernement. Manuel de sophismes politiques, Paris, Bruylant/LGDJ.

BLACK E., 1965, Rhetorical Criticism. A Study in Method, New York, MacMillan.

Boorstin D. J., 1968, An American Primer, New York, Signet (1966, Chicago, University of Chicago Press).

DOMENACH J.-M., 1950, La propagande politique, Paris, PUF (Que sais-je ?)

Ducrot O., 1980, Les échelles argumentatives, Paris, Minuit.

- 1984, Le dire et le dit, Paris, Minuit.

GRIZE J.-B., 1996, Logique et communication, Paris, PUF.

Hochmuтн M., 1955, "The Criticism of rhetoric », A History and Criticism of American

Public Address, vol. III, M. Hochmuth éd., New York, McGraw Hill, p. 1-23.

HAMB LIN C. L., 1970, Fallacies, Londres, Methuen. 
HIRSCHMAN A. O, 1991, Deux siècles de rhétorique réactionnaire [The Rhetoric of Reaction. Perversity, Futility, Jeopardy, trad. P. Andler], Paris, Fayard.

HOFSTADTER R. et al., 1958-1969, Great Issues in American History, vol. I, From Settlement to Revolution, 1584-1776, C. L. Ver Steeg, R. Hofstadter éd., 1958; vol. II, From the Revolution to Civil War, 1765-1865, R. Hofstadter éd., 1958; vol. III, From Reconstruction to the Present Day, 1864-1981, R. Hofstadter, B. K. Hofstadter éd., 19581969, New York, Vintage Books.

KAHANE H., 1971, Logic and contemporary rhetoric, Belmont CA, Wadsworth.

LACOSTE M., 1970, «Éléments de lecture», Recherches rhétoriques. Communications, no 16 , p. 230-235.

Maingueneau D., 1976, Initiation aux méthodes de l'analyse du discours, Paris, Hachette.

MALDIDIER D., 1990, L'inquiétude du discours. Un trajet dans l'histoire de l'analyse du discours : le travail de Michel Pêcheux, textes choisis et présentés par D. Maldidier, Paris, Cendres.

NADEAU R., 1958, «Hermogenes on "stock issues" in deliberative speaking ", Speech Monographs, $\mathrm{n}^{0} 25$ (1), p. 59-66.

Perelman C., Olbrechts-Tyteca L., 1976 [1958], Traité de l'argumentation. La nouvelle rhétorique, Bruxelles, Université de Bruxelles ( $3^{\mathrm{e}}$ édition).

SCHOPEn HAUER A., 1990 [1831], L'art d'avoir toujours raison ou Dialectique éristique, Paris, Circé.

TCHAкотINE S., 1992 [1939], Le viol des foules par la propagande politique, Paris, Gallimard (1re édition en français, censurée, en 1939. Nouvelle édition revue et augmentée, Gallimard, 1952).

TouLMIN S. E., 1993 [1958], Les usages de l'argumentation, Paris, PUF.

VAn Eemeren F. et al., 1987, 1991, 1995, 1999, 2003, 2007, Proceedings (Conferences on Argumentation of the International Society for the Study of Argumentation), Amsterdam, ISSA. 